\title{
An Evolutionary Game approach for the design of congestion control protocols in wireless networks
}

\author{
Eitan Altman ${ }^{\dagger}$, Rachid ElAzouzi*, Yezekeael Hayel*, Hamidou Tembine* \\ * Univesity of Avignon, LIA/CERI, 339, Chemin des Meinajaries, Agroparc BP 1228, F-84911 Avignon \\ $\dagger$ INRIA, Sophia-Antipolis, 2004 Route des Lucioles, F-06902 Sophia-Antipolis
}

\begin{abstract}
Evolutionary games have been developed in biological sciences in the aim of studying the evolution and equilibrium behavior (called Evolutionary Stable Strategies - ESS) of large populations. While rich theoretical foundations of evolutionary games allow biologist to explain past and present evolution and predict future evolution, it can be further used in Engineering to architect evolution. In this paper, we apply evolutionary games to non-cooperative networks containing large number of individual non-cooperative terminals or sensors. We study the evolution of congestion control protocols, and show how the evolution and the ESS are influenced by the characteristics of the wireless channel. We then consider the challenge of architecting the evolution: we propose some guidelines for designing a framework that supports evolution of protocols.
\end{abstract}

\section{INTRODUCTION}

The evolutionary games formalism is a central mathematical tools developed by biologists for predicting population dynamics in the context of interactions between populations. This formalism identifies and studies two concepts: the ESS (for Evolutionary Stable Strategy), and the Replicator Dynamics.

The ESS, defined in 1972 by the biologist Maynard Smith [14], is characterized by a property of robustness against invaders (mutations). More specifically, (i) if an ESS is reached, then the proportions of each population do not change in time. (ii) at ESS, the populations are immune from being invaded by other small populations. This notion is stronger than Nash equilibrium in which it is only requested that a single user would not benefit by a change (mutation) of its behavior. Although ESS has been defined in the context of biological systems, it is highly relevant to engineering as well (see [23]). In the biological context, the replicator dynamics is a model for the change of the size of the population(s) as biologist observe, where as in engineering, we can go beyond characterizing and modeling existing evolution. The evolution of protocols can be engineered by providing guidelines or regulations for the way to upgrade existing ones and in determining parameters related to deployment of new protocols and services. In doing so we may wish to achieve adaptability to changing environments (growth of traffic in networks, increase of speeds or of congestion) and yet to avoid instabilities that could otherwise prevent the system to reach an ESS.

Our first objective is to provide a framework to describe and predict evolution of protocols in a context of competition between two types of behaviors: aggressive and peaceful(see Section III). We use evolutionary games for computing the
ESS for congestion protocols of different degree of aggressiveness. We identify cases in which at ESS, only one population prevails (ESS in pure strategies) and others, in which an equilibrium between several population types is obtained. To study this, we map the problems, whenever possible, into the Hawk and Dove Game. We then study the convergence of the replicator dynamics to it.

The second objective of the paper is to provide a framework for controlling evolutionary dynamics (changing or upgrading protocols) through the choice of a gain parameter governing the replicator dynamics. We address the following two design issues concerning this choice:

(i) the tradeoff between fast convergence and stability. We identify a simple threshold on the gain parameter in the replicator dynamics such that the stability is only determined by whether we exceed or not the threshold.

(ii) the stability as a function of delays. We derive new stability conditions for the replicator dynamics in the Hawk and Dove game with non-symmetric delays and apply it to the evolution of the MAC and transport layer protocols.

The paper is structured as follows. We first provide in the next section the needed background on evolutionary games. We then study the ESS for congestion control protocols (section III). After that, we investigate the impact of the choice of some parameters in the replicator dynamics on the stability of the system in Section IV. Finally we give some numerical investigations and we conclude with concluding remarks.

\section{ESS AND REPLICATOR DYNAMICS}

Consider a large population of players. Each individual needs occasionally to take some action (such as power control decisions, or forwarding decision). We focus on some (arbitrary) tagged individual. Occasionally, the action of some $M$ (possibly random number of) other individuals [18] interact with the action of that individual (e.g. other neighboring nodes transmit at the same time). In order to make use of the wealth of tools and theory developed in the biology literature, we shall often restrict, as they do, to interactions that are limited to pairwise, i.e. to $M=1$. This will correspond to networks operating at light loads, such as sensor networks that need to track some rare events such as the arrival at the vicinity of a sensor of some tagged animal.

We define by $J(p, q)$ the expected payoff for our tagged individual if it uses a strategy $p$ when meeting another individual 
who adopts the strategy $q$. This payoff is called "fitness" and strategies with larger fitness are expected to propagate faster in a population. Note that $J$ is linear in $p$ and $q$.

We assume that there are $N$ pure strategies. A strategy of an individual is a probability distribution over the pure strategies. An equivalent interpretation of strategies is obtained by assuming that individuals choose pure strategies and then the probability distribution represents the fraction of individuals in the population that choose each strategy.

\section{A. Evolutionary Stable Strategies}

Suppose that the whole population uses a strategy $q$ and that a small fraction $\epsilon$ (called "mutations") adopts another strategy $p$. Evolutionary forces are expected to select $q$ against $p$ if

$$
J(q, \epsilon p+(1-\epsilon) q)>J(p, \epsilon p+(1-\epsilon) q)
$$

A strategy $q$ is said to be ESS if for every $p \neq q$ there exists some $\hat{\epsilon}_{y}>0$ such that (1) holds for all $\epsilon \in\left(0, \hat{\epsilon}_{y}\right)$.

In fact, we expect that if for all $p \neq q$,

$$
J(q, q)>J(p, q)
$$

then the mutations fraction in the population will tend to decrease (as it has a lower reward, meaning a lower growth rate). The strategy $q$ is then immune to mutations. If it does not but if still the following holds,

$$
J(q, q)=J(p, q) \text { and } J(q, p)>J(p, p) \quad \forall p \neq q
$$

then a population using $q$ are "weakly" immune against a mutation using $p$ since if the mutant's population grows, then we shall frequently have individuals with strategy $q$ competing with mutants; in such cases, the condition $J(q, p)>J(p, p)$ ensures that the growth rate of the original population exceeds that of the mutants. A strategy is ESS if and only if it satisfies (2) or (3), see [24, Proposition 2.1].

The conditions to be an ESS can be related to and interpreted in terms of Nash equilibrium in a matrix game. The situation in which an individual, say player 1 , is faced with a member of a population in which a fraction $p$ chooses strategy $A$ is then translated to playing the matrix game against a second player who uses mixed strategies (randomizes) with probabilities $p$ and $1-p$, resp. The central model that we shall use to investigate protocol evolution is introduced in the next subsection along with its matrix game representation.

\section{B. The Hawk and Dove (HD) Game}
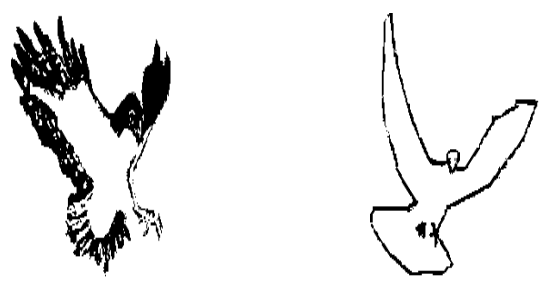

Consider a large population of animals. Occasionally two animals find themselves in competition on the same piece of food. An animal can adopt an aggressive behavior (Hawk) or a peaceful one (Dove). The matrix in Fig. 1 presents the fitness of player I (some arbitrary player) associated with the possible outcomes of the game as a function of the actions taken by each one of the two players. We assume a symmetric game so the utilities of any animal (in particular of player 2) as function of its actions and those of a potential adversary (in particular of player 1), are the same as those player 1 depicted in Figure 1. The utilities (i.e. fitness) represent the following:

An encounter D-D results in a peaceful, equal-sharing of the food which translates to a fitness of 0.5 to each player.

An encounter $\mathbf{H}-\mathbf{H}$ results in a fight in which with equal chances, one or the other player obtains the food but also in which there is a positive probability for each one of the animals to be wounded. Then the fitness of each player is $0.5-\mathrm{d}$, where the 0.5 term is as in the D-D encounter and the $-d$ term represents the expected loss of fitness due to being injured.

An encounter H-D or D-H results in zero fitness to the $\mathrm{D}$ and in one unit of utility for the $\mathrm{H}$ that gets all the food without fight.

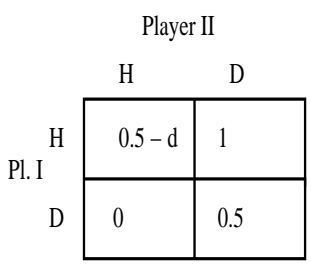

Fig. 1. H-D game in matrix form

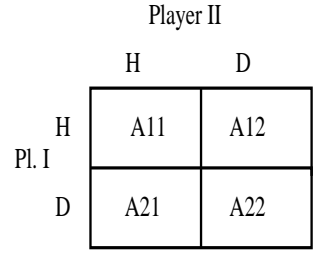

Fig. 2. Generalized H-D game
A more general description of $\mathrm{H}-\mathrm{D}$ games is available in [21].

One can indeed think of other scenarios that are not covered in the original $\mathrm{H}-$ D game, such as the possibility of a Hawk to find the Dove, in a H-D encounter, more delicious than the food

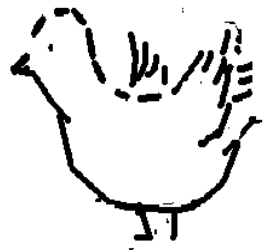
they compete over.

The generalized version [21] of the HD game given in Figure 2 is characterized by $A 11<A 22<A 12$ and $A 21<A 22$. In that case,

1) if $A 11>A 21$ then the pure strategy $H$ is the unique ESS,

2) If $A 11<A 21$ then there is a unique $\operatorname{ESS} p=\left(p_{L}, p_{H}\right)$, it is a mixed strategy given by $p_{H}=u /(u+v)$ where $A i j=J(i, j), i, j \in\{H, D\}, u=A 12-A 22, v=$ $A 21-A 11$.

Remark 2.1: (i) Note that there are no settings of parameters for which the pure strategy D is an ESS in the H-D game 
(or in its generalized version).

(ii) In case 2 above, the strategies $(H, D)$ and $(D, H)$ are pure Nash equilibria in the matrix game. Being asymmetric, they are not candidates for being an ESS according to our definition. There are however contexts in which one obtains non-symmetric ESS, in which case they turn out to be ESS.

\section{Evolution: replicator dynamics}

We introduce here the replicator dynamics [17] which describes the evolution in the population of the various strategies. In the replicator dynamics, the share of a strategy in the population grows at a rate equal to the difference between the payoff of that strategy and the average payoff of the population. More precisely, consider $N$ strategies. Let $\mathbf{x}$ be the $N$ dimensional vector whose $i$ th element $x_{i}$ is the population share of strategy $i$. Thus we have $\sum_{i} x_{i}=1$ and $x_{i} \geq 0$. Below we denote by $J(i, k)$ the expected payoff (or the fitness) for a player using strategy $i$ when it encounters a player with strategy $k$. With some abuse of notation we define $J(i, \mathbf{x})=\sum_{j} J(i, j) x_{j}$. Then the replicator dynamics is defined as

$$
\begin{aligned}
\dot{x}_{i}(t) & =x_{i}(t) K\left(J(i, \mathbf{x}(t))-\sum_{j} x_{j}(t) J(j, \mathbf{x}(t))\right) \\
& =x_{i}(t) K\left(\sum_{j} x_{j}(t) J(i, j)-\sum_{j} \sum_{k} x_{j}(t) J(j, k) x_{k}(t)\right)
\end{aligned}
$$

where $K$ is a positive constant and $\dot{x}_{i}(t):=\mathrm{d} x_{i}(t) / \mathrm{d} t$. Note that the right hand side vanishes when summing over $i$. This is compatible with the fact that we study here the share of each strategy rather than the size of the population that uses each one of the strategies.

\section{Replicator dynamics with delay}

In Equation (4), the fitness of strategy $i$ at time $t$ has an instantaneous impact on the rate of growth of the population size that uses it. An alternative more realistic model for replicator dynamic would have some delay: the fitness acquired at time $t$ will impact the rate of growth $\tau$ time later. We then have

$$
\dot{x}_{i}(t)=x_{i}(t) K\left(\sum_{j} x_{j}(t-\tau) J(i, j)-\sum_{j, k} x_{j}(t) J(j, k) x_{k}(t-\tau)\right)
$$

where $K$ is some positive constant. The delay $\tau$ represents a time scale much slower than the physical (propagation and queueing) delays, it is related to the time scale of (i) switching from the use of one protocol to another (ii) upgrading protocols. More general description of delayed evolutionary game dynamics can be found in [19], [20].

\section{CONGESTION CONTROL PROTOCOLS}

\section{A. Background}

When transferring data between nodes, flow control protocols are needed to regulate the transmission rates so as to adapt to the available resources. A connection that looses data units has to retransmit them later. In the absence of adaptation to the congestion, the on going transmissions along with the retransmissions can cause increased congestion in the network resulting in losses and further retransmissions by this and/or by other connections. This type of phenomenon, that leads to several 'congestion collapses' [8], motivated the evolution of the Internet transport protocol, TCP, to a protocol that reduces dramatically its throughput upon congestion detection.

There are various versions of the TCP protocol among which the mostly used one is New-Reno. The degree of 'aggressiveness' varies from version to version. The behavior of New-Reno is approximately AIMD (Additive Increase Multiplicative Decrease): it adapts to the available capacity by increasing the window size in a linear way by $\alpha$ packets every round trip time and when it detects congestion it decreases the window size to $\beta$ times its value. The constants $\alpha$ and $\beta$ are 1 and $1 / 2$, respectively, in New Reno.

In last years, more aggressive TCP versions have appeared, such as HSTCP (High Speed TCP) [15] and Scalable TCP [9]. HSTCP can be modeled by an AIMD behavior where $\alpha$ and $\beta$ are not constant anymore : $\alpha$ and $\beta$ have minimum values of 1 and of $1 / 2$, resp. and both increase in the window size. Scalable TCP is an MIMD (Multiplicative Increase Multiplicative Decrease) protocol, where the window size increases exponentially instead of linearly and is thus more aggressive. Versions of TCP which are less aggressive than the New-Reno [13] also exist, such as Vegas [5].

Several researchers have analyzed the performance of networks in which various transport protocols coexist, see [1], [3], [4], [10], [16]. In all these papers, the population size using each type of protocol is fixed.

Some papers have already considered competition between aggressive and well behaved congestion control mechanisms within a game theoretic approach. Their conclusions in a wireline context was that if connections can choose selfishly between a well behaved cooperative behavior and an aggressive one then the Nash equilibrium is obtained by all users being aggressive and thus in a congestion collapse [6], [11].

Our approach yields qualitative results, stronger than those obtained through the traditional Nash equilibrium concept adopted in these references. It allows in particular to study the evolution to the equilibrium, and to obtain a sharper characterization of the equilibrium as being robust not only against a single user deviation but also against deviations of a whole (small) fraction of the population.

By casting the problem in our framework of the Hawk and Dove evolutionary game, we shall be able to predict whether a given version of TCP is expected to dominate others (ESS in pure strategies, which means that some versions of TCP would disappear) or whether several versions would co-exist. This would depends also on the network context: an aggressive version of TCP that may dominate in a wireline context may loose its dominance in a wireless network. Indeed, an aggressive TCP may generate higher packet loss rate than other less aggressive versions. These are evaluated more severely in a wireless environment since they represent energy inefficiency which is costly in that environment. 
During the last few years, many researchers have been studying TCP performances in terms of energy consumption and average goodput within wireless networks [13], [25]. Via simulation, the authors show that the TCP New-Reno can be considered as well performing within wireless environment among all other TCP variants and allows for greater energy savings. Indeed, a less aggressive TCP, as TCP New-Reno, may generate lower packet loss than other aggressive TCP. By using the HD game, we show the same behavior of TCP variants.

The model. We consider two populations of connections, all of which use AIMD TCP. A connection of population $i$ is characterized with a linear increase rate $\alpha_{i}$ and a multiplicative decrease factor $\beta_{i}$. Let $x_{i}(t)$ be the transmission rate of connection $i$ at time $t$. We consider the following simple model for competition.

(i) The RTT (round trip times) are the same for all connections.

(ii) There is light traffic in the system in the sense that a connection either has all the resources its needs or it shares the resources with one other connection. (If files are large then this is a light regime in terms of number of connections but not in terms of workload).

(iii) Losses occur whenever the sum of rates reaches the capacity $C: x_{1}(t)+x_{2}(t)=C$.

(iv) Losses are synchronized: when the combined rates attain $C$, both connections suffer from a loss. This synchronization has been observed in simulations for connections with RTTs close to each other [2]. The rate of connection $i$ is reduced by the factor $\beta_{i}<1$.

(v) As long as there are no losses, the rate of connection $i$ increases linearly by a factor $\alpha_{i}$.

We say that a TCP connection $i$ is more aggressive than a connection $j$ if $\alpha_{i} \geq \alpha_{j}$ and $\beta_{i} \geq \beta_{j}$. Let $\bar{\beta}_{i}:=1-\beta_{i}$. Let $x_{n}$ and $y_{n}$ be the transmission rates of connection $i$ and $j$, respectively, just before a loss occurs. We have $x_{n}+y_{n}=C$. Just after the loss, the rates are $\beta_{1} x_{n}$ and $\beta_{2} y_{n}$. The time it takes to reach again $C$ is

$$
T_{n}=\frac{C-\beta_{1} x_{n}-\beta_{2} y_{n}}{\alpha_{1}+\alpha_{2}}
$$

which yields the difference equation:

$$
x_{n+1}=\beta_{1} x_{n}+\alpha_{1} T_{n}=q x_{n}+\frac{\alpha_{1} C \bar{\beta}_{2}}{\alpha_{1}+\alpha_{2}}
$$

where $q=\frac{\alpha_{1} \beta_{2}+\alpha_{2} \beta_{1}}{\alpha_{1}+\alpha_{2}}$. The solution is given by

$$
x_{n}=q^{n} x_{0}+\left(\frac{\alpha_{1} C \bar{\beta}_{2}}{\alpha_{1}+\alpha_{2}}\right) \frac{1-q^{n}}{1-q} .
$$

\section{B. HD game: throughput-loss tradeoff}

In wireline, the utility related to file transfers is usually taken to be the throughput, or a function of the throughput (e.g. the delay). It does not explicitly depend on the loss rate. This is not the case in wireless context. Indeed, since TCP retransmits lost packets, losses present energy inefficiency. Since energy is a costly resource in wireless, the loss rate is included explicitly in the utility of a user through the term representing energy cost. We thus consider fitness of the form $J_{i}=T h p_{i}-\lambda R$ for connection $i$; it is the difference between the throughput $T h p_{i}$ and the loss rate $R$ weighted by the so called tradeoff parameter, $\lambda$, that allows us to model the tradeoff between the valuation of losses and throughput in the fitness. We now proceed to show that our competition model between aggressive and non-aggressive TCP connections can be formulated as a HD game. We study how the fraction of aggressive TCP in the population at (the mixed) ESS depends on the tradeoff parameter $\lambda$.

Since $|q|<1$, we get the following limit $x$ of $x_{n}$ when $n \rightarrow \infty$ :

$$
x=\frac{\alpha_{1} C \bar{\beta}_{2}}{\alpha_{1}+\alpha_{2}} \cdot \frac{1}{1-q}=\frac{\alpha_{1} \bar{\beta}_{2} C}{\alpha_{1} \bar{\beta}_{2}+\alpha_{2} \bar{\beta}_{1}} .
$$

It is easily seen that the share of the bandwidth (just before losses) of a user is increasing in its aggressiveness. Hence the average throughput of connection 1 is

$$
T h p_{1}=\frac{1+\beta_{1}}{2} \times \frac{\alpha_{1} \bar{\beta}_{2}}{\alpha_{1} \bar{\beta}_{2}+\alpha_{2} \bar{\beta}_{1}} \times C .
$$

The average loss rate of connection 1 is the same as that of connection 2 and is given by

$$
R=\frac{1}{T}=\left(\frac{\alpha_{1}}{\bar{\beta}_{1}}+\frac{\alpha_{2}}{\bar{\beta}_{2}}\right) \frac{1}{C} \text { where } T=\frac{\bar{\beta}_{1} \bar{\beta}_{2} C}{\alpha_{1} \bar{\beta}_{2}+\alpha_{2} \bar{\beta}_{1}}
$$

with $T$ being the limit as $n \rightarrow \infty$ of $T_{n}$.

Let $\mathrm{H}$ corresponds to $\left(\alpha_{H}, \beta_{H}\right)$ and $\mathrm{D}$ to $\left(\alpha_{D}, \beta_{D}\right)$ such that $\alpha_{H} \geq \alpha_{D}$ and $\beta_{H} \geq \beta_{D}$. Then, for $i=1,2, T h p_{i}(H, H)=$ $\operatorname{Thp}_{i}(D, D)$. Since the loss rate for any user is increasing in $\alpha_{1}, \alpha_{2}, \beta_{1}, \beta_{2}$ it then follows that $J(H, H)<J(D, D)$, and $J(D, H)<J(D, D)$. We conclude that the utility that describes a tradeoff between average throughput and the loss rate leads to the HD structure.

The mixed ESS is given by the following probability of using $\mathrm{H}$ :

$$
x^{*}(\lambda)=\frac{\eta_{1}-\eta_{2} \lambda}{\eta_{3}} \quad \text { where }
$$

$$
\begin{gathered}
\eta_{1}=\left(\bar{\mu} \frac{1+\beta_{1}}{2}-\frac{1+\beta_{2}}{4}\right) C, \eta_{2}=\frac{1}{C}\left(\frac{\alpha_{1}}{\bar{\beta}_{1}}-\frac{\alpha_{2}}{\bar{\beta}_{2}}\right), \\
\eta_{3}=C\left(\frac{1}{2}-\mu\right) \frac{\beta_{1}-\beta_{2}}{2}, \mu=\frac{\alpha_{2}\left(\bar{\beta}_{1}\right)}{\alpha_{2}\left(\bar{\beta}_{1}\right)+\alpha_{1}\left(\bar{\beta}_{2}\right)} .
\end{gathered}
$$

where $\bar{\mu}:=1-\mu$. Note that $\eta_{2}$ and $\eta_{3}$ are positive. Hence, the equilibrium point $x^{*}$ decrease linearly on $\lambda$. We conclude that applications that are more sensitive to losses would be less aggressive at ESS.

\section{Tradeoff between transient and steady-state behavior}

When the available bandwidth along the route of a connection changes due to new cross-traffic or due to failures, an aggressive connection could take much longer to adapt to 
the available bandwidth than other connections. It would thus suffer from more losses and during a longer period than if it were less aggressive. This would mean more retransmissions and longer times needed to send files in the case of transient decrease of available bandwidth. In wireless networks, this would also mean larger energy consumption. We may thus expect aggressive versions of transport protocols to have lower fitness in wireless networks than in wireline ones. We sketch some steps that can be followed in modeling the above scenario.

Assume that there is a single connection such that, at time 0 , it has the full available throughput $C_{\max }$. Assume that the available bandwidth decreases to $\gamma C_{\max }$ for some $\gamma<1$. Define $T_{n}=n \times R T T$ and assume that for each integer $n$, the transmission rate decreases at $T_{n}$ by a factor of $\beta$ if there have been one or more losses during the interval $\left[T_{n-1}, T_{n}\right)$ (This feature is inspired by the behavior of the New Reno version of TCP). Then it can be shown that the time it takes the protocol to reach the transmission rate of $\gamma C_{\max }$ is $m \times R T T$, where $m$ is given (approximately) by $m=\frac{\log (\gamma)}{\log (\beta)}$. Till time $m \times R T T$, the goodput cannot exceed $\gamma C_{\max }$ so the loss rate $\lambda_{t}$ at $t \in[0, m \times R T T]$ is at least the difference between the transmission rate and $\gamma C_{\max }$. The number of losses during that period is then $\int_{0}^{m \times R T T} \lambda_{t} d t$.

\section{ARCHITECTING EVOLUTION}

We study the choice of two parameters in the replicator dynamics that impact the stability of the evolution process of protocols: the gain parameter $K$ and the delay $\tau$ appearing in Equation (5). The standard replicator dynamics (4) appearing in the evolutionary game literature is defined with $K=1$. $K$ 's other than one can be interpreted as if the utilities $J$ are multiplied by a constant. Alternatively, it can be seen as scaling time. The parameter $K$ can thus be used to accelerate the rate of convergence in (4).

\section{A. The impact of $K$ and $\tau$ on the stability}

We consider below the case of two players and two actions. Define

$$
\begin{gathered}
\delta_{1}=J(B, A)-J(A, A), \quad \delta_{2}=J(A, B)-J(B, B), \\
\delta=\delta_{1}+\delta_{2}, \quad \theta=\frac{\delta \pi}{2 \delta_{1} \delta_{2}}
\end{gathered}
$$

Guidelines for an evolution framework. For $K=1$, it has been shown in [21] that if the delay $\tau$ in (5) satisfies $\tau<\theta$ then the mixed ESS (given in section II-B) is asymptotically stable, and if $\tau$ increases beyond $\theta$ then the ESS becomes unstable.

We make the observation that Equation (5) with $K$ is equivalent to Equation (4) with all elements $J(i, m)$ multiply by $K$. Thus we can use the result of [21] to conclude that the stability condition for general $K$ is given simply by

$$
\tau K<\theta
$$

This provides us with an important guideline for designing evolutionary protocols. In order for such a protocol to be scalable to any delay, the product of the adaptation speed parameter $K$ and delay $\tau$ should be $O(1)$. Thus the larger the delay is, the slower we should react to the fitness of a strategy being used.

We note that this type of scaling is quite familiar in other networking contexts: the internet transport protocol TCP has a throughput that scales according to 1/RTT (where RTT is the round trip delay). This scaling is obtained by a self clocking mechanism based on ACKs that trigger new transmissions.

\section{B. Numerical investigation}

Impact of gain parameter Our first numerical experiment studies the behavior of the replicator dynamics for the case of one delay unit as a function of $K$ : we check the speed of convergence and the stability of the replicator dynamics as a function of the gain parameter $K$. We consider the following fixed parameters: we took $\tau=1, \delta=2 / 3$, and let $K$ vary between 0.16 and 15. A unique mixed ESS exists for these parameters, for which the fraction of the population using $\mathrm{H}$ is $3 / 4$.

The resulting trajectories of the population ratio using the first strategy, $H$, as a function of time, is given in Fig. 3 Top. For $K=0.16$, we have stability but the convergence speed is slow. The other extreme is illustrated for $K=15$ which is seen to be unstable: it oscillates rapidly and the amplitude is seen to grow slowly.

Impact of delay We now keep $K$ constant and evaluate the stability varying the delay between 0.016 and 15 time units. When $\tau=0.016$ the system is stable but the rate of convergence to the interior equilibrium is not fast. For $\tau=15$ the system is unstable, the solution oscillates around the equilibrium $x^{*}=3 / 4$.

Oscillating Solution and dependence on the initial state. In Figure 3 (Bottom) we display an oscillatory behavior of the population ratios as function of time for two different initial values of $x(0)=0.03$ and $x(0)=0.97$ with $K=15$ and $\tau=1$. It corresponds to an unstable regime in which the ESS is not attained. The trajectories are seen to converge to periodic ones. The limit trajectories look the same and do not depend on the initial state except for a dependence through the phase. In this unstable regime, more than one protocol coexist and the ratio of population sizes using the protocols has oscillations with large amplitude.

Validation of stability conditions. In both Top and Middle parts of Figure 3, we observe that we have stability when $\tau K<4 \pi \simeq 12.56$. Indeed, in top part of figure 3 , the parameter $\tau=1$, hence the condition of stability (6) becomes $K<12.56$. This actually confirm that using $K=0.16,1,1.56,12$, the system is stable and using $K=15$, the system is unstable. We observe the same behavior when keeping $K$ constant and varying the delay $\tau$. 

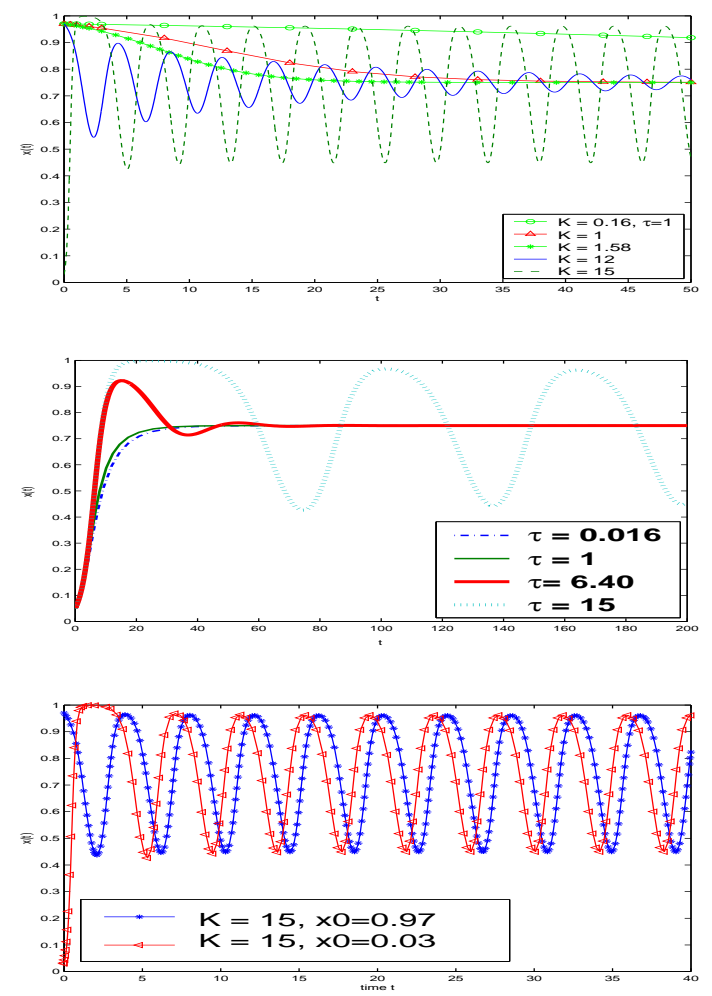

Fig. 3. Top: Effect of $\mathrm{K}$ on stability. Middle: Effect of $\tau$ on stability. Bottom: Effect of initial state

\section{CONCLUding REMARKS}

In this paper, we have studied evolutionary aspects of protocols in wireless communications using the biological paradigm of evolutionary games. Wireless networking has yet other aspects in common with biology, both behavioral, such as the distributed nature of (possibly non-cooperative) decision making, as well as physical, such as the crucial dependence on energy resources and hence the need for energy conservation policies [12]. We plan to further follow biologists [7] in studying the combination of these issues within a dynamic evolutionary framework. We shall study the advantages and disadvantages for protocols to behave more (or less) aggressively when there is little energy remaining, so as to predict the type of protocols that would prevail in an evolutionary competitive environment. In the full version of this paper, we give some remarks on cooperation and noncooperative behaviors.

\section{ACKNOWLEDGEMENT}

This work was partially supported by the BIONETS European Contract. We thank two anonymous reviewers for their many suggestions for improving this paper.

\section{REFERENCES}

[1] O Ait-Hellal and E. Altman. "Analysis of TCP Vegas and TCP Reno",

Telecommunication Systems, 15:381-404, 2000.
[2] O. Ait-Hellal, E. Altman, D. Elouadghiri, M. Erramdani, N. Mikou, "Performance of TCP/IP: the case of two Controlled Sources", ICCC'97, Cannes, France, November 19-21, 1997.

[3] E. Altman, K. Avrachenkov, and B. Prabhu. "Fairness in MIMD congestion control algorithms", Telecommunication Systems, 30(4): $387-415$.

[4] T. Bonald, "Comparison of TCP Reno and TCP Vegas: Efficiency and Fairness", Perform. Eval. 36-37(1-4): 307-332 (1999).

[5] Brakmo L.S., O'Malley S., and L.L. Peterson. 'Tcp vegas: New techniques for congestion detection and avoidance", Computer Communication Review, Vol. 24, No. 4, pp. 24-35, Oct., 1994.

[6] R. Garg, A. Kamra, V. Khurana, A game-theoretic approach towards congestion control in communication networks SIGCOMM Computer Communication Review archive 32(3) (July 2002) 47 - 61.

[7] A. I. Houston and J. M. McNamara, "Evolutionarily stable strategies in the repeated hawk-dove game", Behavioral Ecology, pp. 219-227, 1991.

[8] Van Jacobson "Congestion Avoidance and Contrnol", SIGCOMM '88, Stanford, CA, Aug. 1988.

[9] T. Kelly, "Scalable TCP: Improving Performance in Highspeed Wide Area Networks". Computer Comm. Review 32(2), April03.

[10] L. Lopez, A. Fernandez and V. Cholvi. "A Game Theoretic Analysis of Protocols Based on Fountain Codes". IEEE ISCC'2005, June 2005

[11] L. Lopez, G. Rey, A. Fernandez and S. Paquelet, "A mathematical model for the TCP tragedy of the commons", Theoretical Computer Science, 343, pp. 4-26, 2005.

[12] V. Mhatre and C. Rosenberg, "Energy and cost optimizations in wireless sensor networks: A survey," 25th Anniversary of GERAD, Kluwer Academic Publishers, Jan 2004.

[13] H. Singh, S. Singh, "Energy consumption of TCP Reno, New Reno and SACK in multi-hop wireless networks, ACM SIGMERTICS, Jun. 2002.

[14] M. Smith, "Game Theory and the Evolution of Fighting." In John Maynard Smith, On Evolution (Edinburgh University Press), pp.8-28, 1972.

[15] E. Souza and D.A. Agarwal. "A HighSpeed TCP study: Characteristics and deployment issues", Technical Report LBNL-53215, Lawrence Berkeley National Laboratory, 2002.

[16] A. Tang, J. Wang, S. Low, and M. Chiang. "Equilibrium of heterogeneous congestion control: Existence and uniqueness", IEEE/ACM Transactions on Networking, Oct 2007.

[17] P.D. Taylor and L. Jonker, "Evolutionarily stable strategies and game dynamics," Math. Bioscience, vol.40, pp.145-156, 1978.

[18] H. Tembine, E. Altman, R. El-Azouzi and Y. Hayel, Evolutionary games with random number of interacting players with application to access control, to appear in Proc. of WiOpt'08.

[19] H. Tembine, E. Altman and R. ElAzouzi, Asymmetric Delay in Evolutionary Games, VALUETOOLS, Oct. 2007.

[20] H. Tembine, E. Altman and R. El-Azouzi. Delayed Evolutionary Game Dynamics applied to the Medium Access Control, in Proc. of Bionetworks, Oct. 2007.

[21] Tao, Y. and Wang, Z., 1997. "Effect of time delay and evolutionarily stable strategy", J. Theor. Biol. 187, 111-116.

[22] Eric van Damme, Stability and perfection of Nash equilibria, Springer, Berlin, 1991.

[23] T. L. Vincent and T. L. S. Vincent, "Evolution and control system design”, IEEE Control Systems Magazine, Vol 20 No. 5, pp 20-35, Oct. 2000.

[24] Weibull J., (1995) Evolutionary Game Theory, M.I.T. Press.

[25] M. Zorzi and R. Rao, "Energy efficiency of TCP in a local wireless environment, Mobile Networks and Applications, Vol. 6, No. 3,2001. 